\title{
Neutron capture reactions on Lu isotopes at DANCE
}

\author{
O. Roig ${ }^{1,2, a}$, M. Jandel ${ }^{2}$, D.J. Vieira ${ }^{2}$, E.M. Bond ${ }^{2}$, T.A. Bredeweg ${ }^{2}$, A.J. Couture $^{2}$, J-M. Daugas ${ }^{1}$, R.C Haight ${ }^{2}$, A.L. \\ Keksis $^{2}$, V. Méot ${ }^{1}$, P. Morel ${ }^{1}$, J.M. O’Donnell' ${ }^{2}$, R.S. Rundberg ${ }^{2}$, W.A. Taylor ${ }^{2}$, J.L. Ullmann ${ }^{2}$, and J.M. Wouters ${ }^{2}$ \\ 1 CEA DAM DIF, F-91297 Arpajon, FRANCE \\ 2 Los Alamos National Laboratory, Los Alamos, New Mexico 87545, USA
}

\begin{abstract}
The DANCE (Detector for Advanced Neutron Capture Experiments) array located at the Los Alamos national laboratory has been used to obtain the neutron capture cross sections for the ${ }^{175} \mathrm{Lu}$ and ${ }^{176} \mathrm{Lu}$ isotopes with neutron energies from thermal up to $100 \mathrm{keV}$. Both isotopes are of current interest for the nucleosynthesis s-process in astrophysics and for applications as in reactor physics or in nuclear medicine. Three targets were used to perform these measurements. One was ${ }^{n a t} \mathrm{Lu}$ foil and the other two were isotope-enriched targets of ${ }^{175} \mathrm{Lu}$ and ${ }^{176} \mathrm{Lu}$. The cross sections are obtained for now through a precise neutron flux determination and a normalization at the thermal neutron cross section value. A comparison with the recent experimental data and the evaluated data of ENDF/B-VII.0 will be presented. In addition, resonances parameters and spin assignments for some resonances will be featured.
\end{abstract}

\section{Introduction}

Neutron capture cross sections are of current interest in nuclear astrophysics and for the nuclear reaction models. Nuclear data for both topics are crucial to improve the predictive capabilities of the models. On one hand, the synthesis of heavy elements is dominated by neutron induced reactions. The thermonuclear reaction rates are critical for understanding these reactions. The reaction rates needed for s-process nucleosynthesis are particularly amenable to experimental investigation as the s process follows the valley of beta-stability, making most of the reactions of interest take place on stable isotopes. ${ }^{175} \mathrm{Lu}$ is an important waiting point while ${ }^{176} \mathrm{Lu}$ exhibits a thermally enhanced beta decay rate, making it a sensitive branch point, both for estimating neutron densities as well as temperatures at the nucleosynthesis site $[1,2]$. Typically, the neutron capture reaction rates are obtained by measuring the neutron capture cross section using a neutron spectrum similar to a Maxwell-Boltzmann distribution at a given stellar temperature [3]. To get a complete set of such data between $5 \mathrm{keV}$ and $100 \mathrm{keV}$ stellar temperatures, we need to use cross sections for a neutron energy range as wide as possible. On the other hand, this kind of experiments provides also the opportunity to further constraint reaction models obtaining nuclear structure information like resonance spin assignement and level density [4] or testing the gammarays strenght functions $[5,6]$. To check the predictive capabilities of nuclear reaction models, we have envisaged to pursue performing such experiments on some Lu unstable isotopes as the ${ }^{173} \mathrm{Lu}$.

By combining the capabilities of the Los Alamos Neutron Scattering CEnter (LANSCE) accelerators and the De-

\footnotetext{
a e-mail: olivier.roig@cea.fr
}

tector for Advanced Neutron Capture Experiments (DANCE) array, neutron capture measurements on the ${ }^{175} \mathrm{Lu}$ and ${ }^{176} \mathrm{Lu}$ isotopes have been undertaken from thermal neutron energy to few hundred $\mathrm{keV}$ using isotope enriched targets. Here we report on the determination of the neutron capture cross sections on the ${ }^{175} \mathrm{Lu}$ and ${ }^{176} \mathrm{Lu}$ isotopes and the resonance characterisation using a R-matrix code.

\section{Experimental setup}

\subsection{The DANCE detector}

The DANCE detector at the Los Alamos Neutron Science Center (LANSCE) flight path is located at $20 \mathrm{~m}$ from the white neutron source of the Lujan Center. This neutron production is based on spallation reactions on a tungsten target. The DANCE detector consists of $160 \mathrm{BaF}_{2}$ crystals in a $4 \pi$ geometry. These crystals detect gamma-rays following neutron capture with high efficiency about $86 \%$ at $1 \mathrm{MeV}$. The efficiency of gamma cascade is typically around $98 \%$. Its high segmentation enables gamma multiplicity measurements and high counting rate. The fast timing allows precise gamma-gamma coincidence and time of flight measurements. Small or radioactive samples can be used. The acquisition system uses the technique of digitization to maximize the data available from the detector. For that, DANCE acquisition has high speed digitizer cards at 500 MSamples/s (1 point each 2 ns), managed by 14 frontend computers. An on-line analysis is performed to store reduced waveform consisting in the integrals of the slow and fast signal components, times and multiplicities for an acquired event [7].

This is an Open Access article distributed under the terms of the Creative Commons Attribution-Noncommercial License, which permits unrestricted use, distribution, and reproduction in any noncommercial medium, provided the original work is properly cited. 
Table 1. Characteristics of Lu targets used at DANCE.

\begin{tabular}{|c|c|c|c|c|}
\hline Target & Diameter & Backing & Purity & $\begin{array}{l}\text { Mass } \\
\left(\mathrm{mg} / \mathrm{cm}^{2}\right)\end{array}$ \\
\hline${ }^{n a t} \mathrm{Lu}$ & $25 \mathrm{~mm}$ & self-supported & $99.90 \%$ & 31 \\
\hline${ }^{175} \mathrm{Lu}$ & $6.35 \mathrm{~mm}$ & $\begin{array}{l}2.5 \mu \mathrm{m} \text { tita- } \\
\text { nium foils }\end{array}$ & $99.80 \%$ & 1 \\
\hline${ }^{176} \mathrm{Lu}$ & $7 \mathrm{~mm}$ & $\begin{array}{l}1 \mu \mathrm{m} \quad \text { alu- } \\
\text { minized mylar }\end{array}$ & $99.95 \%$ & 1 \\
\hline${ }^{173} \mathrm{Lu}$ & $6.35 \mathrm{~mm}$ & $\begin{array}{l}2.5 \mu \mathrm{m} \text { tita- } \\
\text { nium foils }\end{array}$ & $16 \%$ & $21 \times 10^{-3}$ \\
\hline
\end{tabular}

\subsection{The Lu targets}

Four targets were used to perform these measurements. Characteristics of these targets are reported in Table 1. The ${ }^{n a t}$ Lu target was a metallic foil. The electrodeposition technique has been used for most of these isotopically enriched targets except the ${ }^{176} \mathrm{Lu}$ target for which a mass separator [8] was used to extract and deposit the ${ }^{176} \mathrm{Lu}$ isotope.

A ${ }^{173} \mathrm{Lu}$ target was exposed to the neutron beam at DANCE. This radioactive target of ${ }^{173} \mathrm{Lu}$ was produced at the Isotope Production Facility in Los Alamos following by a hot chemical separation.

\subsection{Data analysis}

Each experiment have taken 10 days of beam. The goal of the data analysis was to get the cross section $\sigma_{(n, \gamma)}$ according to this formulae 1

$$
\sigma_{n, \gamma}\left(E_{n}\right)=\frac{M}{N_{A} \times \rho_{S}} \times \frac{N_{(n, \gamma)}\left(E_{n}\right)}{\epsilon\left(E_{n}\right) \phi\left(E_{n}\right) S}
$$

where $\mathrm{N}_{(n, \gamma)}$ is the yield of the neutron capture events at one neutron energy bin. To determine the absolute cross section, the detection efficiency $\epsilon$ was extracted from the simulation code GEANT4 [9] using an input file from a gamma cascade code [10]. Some cuts were applied on our data. To correct these selections, the cut efficiencies have been determined. $\mathrm{A} \mathrm{BF}_{3}$ beam monitor was used to get the neutron flux $\phi\left(E_{n}\right)$. This flux was normalized using a gold sample placed at the target position. The data analysis for a DANCE experiment is well described in reference [11]. Following results presented in this paper are normalized cross sections at $\mathrm{E}_{n}=0.0253 \mathrm{eV}$ from the reference [12].

\section{Results and discussion}

\subsection{The ${ }^{176} \mathrm{Lu}$ isotope}

The results on ${ }^{176} \mathrm{Lu}(\mathrm{n}, \gamma)$ cross section are shown in Fig. 3.1 and 2. The R-matrix SAMMY code [14] is used to fit the experimental data using the ENDF/B-VII.0 evaluations as initial resonance parameters. The resonance energies, $\Gamma_{\gamma}$ and $\Gamma_{n}$ widths were free parameters for all resonances. A very good agreement with DANCE data is found in the resonance region. Figure 3.1 shows that the background

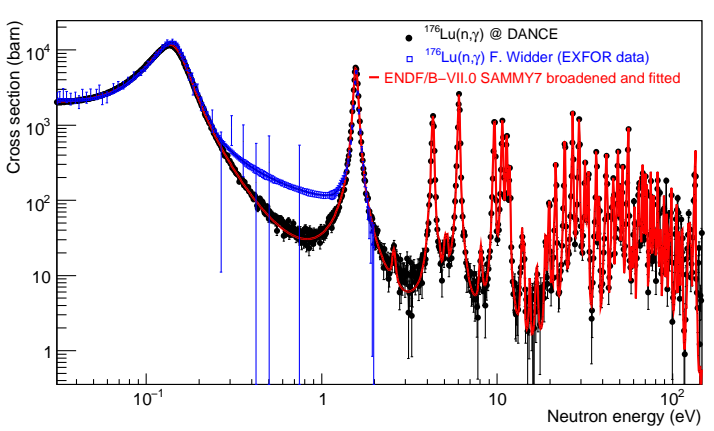

Fig. 1. Neutron capture cross section on ${ }^{176} \mathrm{Lu}$ in the neutron energy range between $0.02 \mathrm{eV}$ and $140 \mathrm{eV}$ measured with the DANCE array (black circles). Red line represents a SAMMY fit using the ENDF/B-VII.0 evaluated data base. Blue markers represents existing experimental data from $\mathrm{F}$. Widder (EXFOR data).

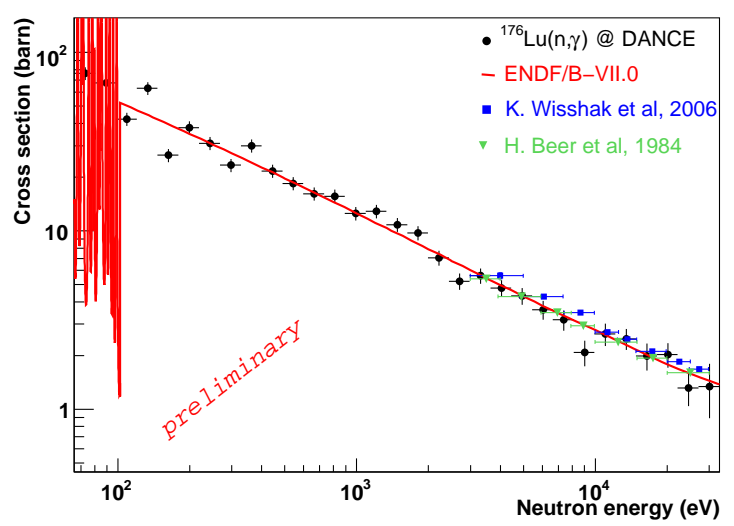

Fig. 2. Neutron capture cross section on ${ }^{176} \mathrm{Lu}$ in the neutron energy range between $100 \mathrm{eV}$ and $100 \mathrm{keV}$ measured with the DANCE array (black circles). Red line represents evaluated data from ENDF/B-VII.0 data base. Blue and green markers represent existing experimental data from $\mathrm{H}$. Beer et al. [16] and K. Wisshak et al. [3] respectively.

subtraction was very efficient in the resonance region. Table 2 presents some resonances parameters extracted from the SAMMY code.

Figure 2 shows the cross section measured at higher energy with the evaluated data from ENDF/B-VII.0. DANCE cross section only normalized at the thermal neutron energy is in good agreement with previous data from $\mathrm{H}$. Beer [16], K. Wisshak [3] and the evaluated cross sections.

Considering only s-wave resonances, the compound nucleus formed by the $(\mathrm{n}, \gamma)$ reaction on the $\mathrm{J}^{\pi}=7^{-}$ground state of the ${ }^{176} \mathrm{Lu}$ can have resonance spins of $13 / 2$ or $15 / 2$. A preliminary study of the averaged $\gamma$ multiplicity distribution shows that we are able to distinguish between spins of s-wave resonances. Figure 3 shows the difference of the averaged multiplicity. Spins indicated in Fig. 3 are from the reference [12]. The spins of unassigned resonance could be attributed. At higher neutron energies, methods proposed by S. Sheets et al. [4] and P. Koelher et al. [17] should be 
$\mathrm{CNR} * 09$

Table 2. Some preliminary resonance parameters for ${ }^{176} \mathrm{Lu}(\mathrm{n}, \gamma)$

\begin{tabular}{ccc|ccc}
\hline \multicolumn{3}{c|}{ Mughabghab (2006) } & \multicolumn{3}{c}{ This work } \\
\hline Energy (eV) & $\Gamma_{\gamma}(\mathrm{meV})$ & $\Gamma_{n}(\mathrm{meV})$ & Energy $(\mathrm{eV})$ & $\Gamma_{\gamma}(\mathrm{meV})$ & $2 \mathrm{~g} \Gamma_{n}(\mathrm{meV})$ \\
\hline $9.73(3)$ & - & $1.370(12)$ & $9.67(7)$ & $66(7)$ & $1.34(14)$ \\
$10.79(4)$ & $72(2)$ & $1.600(17)$ & $10.74(8)$ & $78(8)$ & $1.71(17)$ \\
$11.44(4)$ & $68(8)$ & $1.072(73)$ & $11.40(7)$ & $63(6)$ & $1.01(10)$ \\
$11.88(5)$ & $69(8)$ & $0.442(15)$ & $11.83(17)$ & $61(6)$ & $0.39(4)$ \\
\hline
\end{tabular}

more accurate and would be appropriate to apply for $\mathrm{Lu}$ isotopes data.

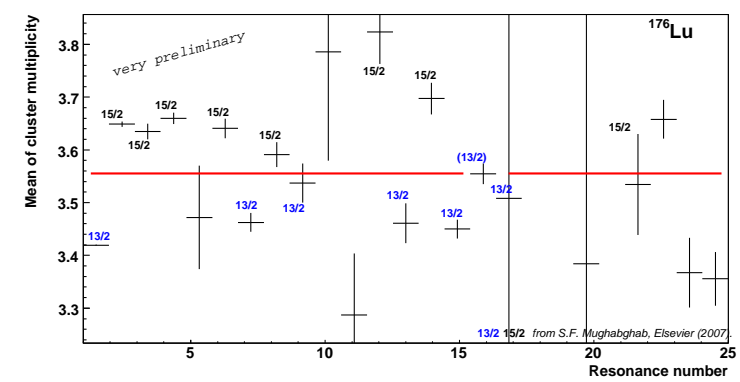

Fig. 3. Average multiplicity for the $25 \mathrm{st}$ resonances of the ${ }^{176} \mathrm{Lu}(\mathrm{n}, \gamma)$ reaction. Black and blue values are spins of s-wave resonances known from Mughaghab [12].

\subsection{The ${ }^{175} \mathrm{Lu}$ isotope}

Figure 4 shows cross sections concerning the natural $\mathrm{Lu}$ neutron capture reaction. As the natural Lu isotopes is mostly compound of the ${ }^{175} \mathrm{Lu}$ isotope $(97.41 \%)$, cross section could be extract for the ${ }^{175} \mathrm{Lu}(\mathrm{n}, \gamma)$ reaction. Corrections should be necessary to subtract contribution from ${ }^{176} \mathrm{Lu}$ and ${ }^{181} \mathrm{Ta}$. ${ }^{181} \mathrm{Ta}$ was found as pollutant of the natural foil. However, the SAMMY code can determine the proportion of each constituant and fit the measured cross section. There is a good agreement between DANCE data and fits from SAMMY using the ENDF/B-VII.0 evaluated data base.

\subsection{Maxwellian-averaged $(\mathbf{n}, \gamma)$ cross sections on ${ }^{176} \mathbf{L u}$}

Since cross sections have been measured over a wide energy range, the MACS can be extracted using the DANCE data. The Maxwellian-averaged $(\mathrm{n}, \gamma)$ cross sections in a stellar plasma of thermal energy kT is defined as in reference [13]. MACS can be defined as a sum of three integrals. The first integral $I_{1}$ is extracted from these DANCE data according to the formula 2 between thermal energy and $3 \mathrm{keV}$ including the resonance region. The other two integrals, $I_{2}$ and $I_{3}$, are taken from reference [3].

$$
I_{1}=\frac{2}{\sqrt{(} \pi)} \frac{1}{(k T)^{2}} \sum \sigma_{n, \gamma} E_{i} \times e^{-E_{i} / k T} \Delta E_{i}
$$

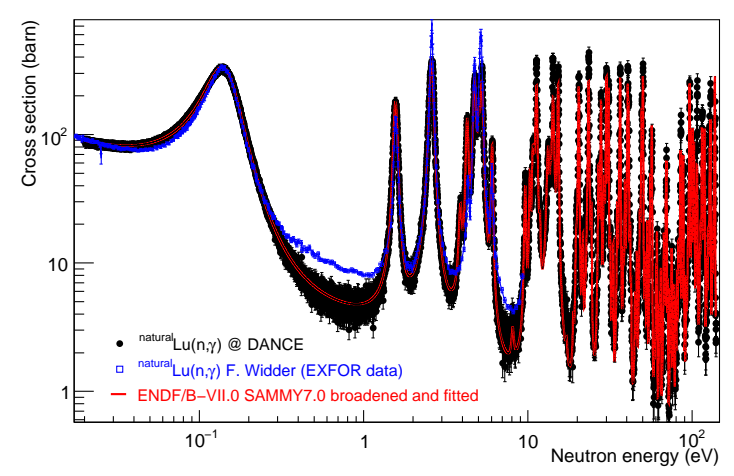

Fig. 4. Neutron capture cross section on ${ }^{n a t} \mathrm{Lu}$ in the neutron energy range between $0.02 \mathrm{eV}$ and $140 \mathrm{eV}$ measured with the DANCE array (black circles). Red line represents a SAMMY fit using the ENDF/B-VII.0 evaluated data base. Blue markers represents existing experimental data from EXFOR data base.

Table 3. Maxwellian-averaged cross section given in mb for various $\mathrm{kT}$ values for ${ }^{176} \mathrm{Lu}(\mathrm{n}, \gamma)$ reaction.

\begin{tabular}{cccc}
\hline kT $(\mathrm{keV})$ & ENDF/B-VII.0 & This work $(\mathrm{mb})$ & Ref.[3] \\
\hline 8 & 3243 & $3487 \pm 52$ & $3586 \pm 62$ \\
10 & 2829 & $3041 \pm 38$ & $3109 \pm 44$ \\
15 & 2230 & $2383 \pm 25$ & $2421 \pm 27$ \\
20 & 1903 & $2024 \pm 19$ & $2046 \pm 20$ \\
25 & 1694 & $1791 \pm 16$ & $1806 \pm 17$ \\
30 & 1548 & $1629 \pm 14$ & $1639 \pm 14$ \\
\hline
\end{tabular}

The calculated MACS for the ${ }^{176} \mathrm{Lu}(\mathrm{n}, \gamma)$ reaction at various thermal energies $\mathrm{kT}$ is given in Table 3 as well the recent values from Wisshak et al. [3]. Resonances have an increasing contribution to the MACS at low temperatures.

\subsection{Conclusion}

The DANCE detector provides us a very complete data set for the neutron capture study that can be used to improve reaction models. We can directly compare our results to the reaction models results in the $\mathrm{keV}$ region. In the resonance region, we are able to obtain valuable nuclear structure information from spin assignments. All of these results are relevant to fundamental nuclear properties and important input parameters for both reaction models and nuclear astrophysics. For the first time, we have obtained over a large neutron range the neutron capture cross sections on the ${ }^{176} \mathrm{Lu}$ and the ${ }^{175} \mathrm{Lu}$ isotopes. A very good agreement between the evaluated data for the ${ }^{176} \mathrm{Lu}$ and the ${ }^{175} \mathrm{Lu}$ and measurements is found. 


\section{Acknowledgements}

We would like to thank Milan Krtička of Charles University, Prague, for his help in using the code DICEBOX. This work has benefited from the use of the Lujan Center at the Los Alamos Neutron Science Center, funded by the DOE Office of Basic Energy Sciences and Los Alamos National Laboratory funded by the Department of Energy under contract W-7405-ENG-36.

\section{References}

[1] K. Takahashi and K. Yokoi, At. Data Nucl. Data Tables 36, p.375 (1987).

[2] N. Klay, F. Käppeler, H. Beer, and G. Schatz, Phys. Rev. C 44, 2839 (1991).

[3] K. Wisshak, F. Voss, F. Käppeler, L. Kazakov Phys. Rev. C 73, 015807 (2006).

[4] S. A. Sheets et al., Phys. Rev. C 76, 064317 (2007).

[5] S. A. Sheets et al., Phys. Rev. C 79, 024301 (2009).

[6] D. Dashdorj et al., in proceedings of the Twentieth International Conference of application of accelerators in research and industry, AIP Conf. Proc. 1099, p.704 (2009).

[7] J.M. Wouters et al., IEEE Transactions on nuclear science, Vol. 53, 3 (2006).

[8] N. Chauvin, F. Dayras, D. Le Du, and R. Meunier, Nucl. Instrum. and Meth. A 521, pp. 149-155 (2004).

[9] S. Agostinelli et al., Nucl. Instr. and Meth. A 506, pp. 250-30 3(2003).

[10] F. Bec̃vàr, Nucl. Instr. and Meth. A 417, p.434 (1998).

[11] M. Jandel et al., Phys. Rev. C 78, 034609 (2008).

[12] S. F. Mughabghab, Atlas of neutron resonances, Elsevier Science (2007).

[13] R.L. Macklin and J.H. Gibbons, Rev. Mod. Phys. 37, 166 (1965).

[14] N. M. Larson, SAMMY, ORNL/TM-9179/7 (2006).

[15] S.M. Kalebin et al., Atomnaya Energiya, 42, Iss. 6, p. 506 (1977)

[16] H.Beer, G.Walter, R.L.Macklin, P.J.Patchett, Physical Review C 30, p.464 (1984)

[17] P. E. Koehler et al., Phys. Rev. C 76, 025804 (2007). 